\title{
Spotlights on some electrocardiographic paradigms: How should we evaluate normal reference values of Tp-Te interval, Tp-Te dispersion and Tp-Te/QT ratio?
}

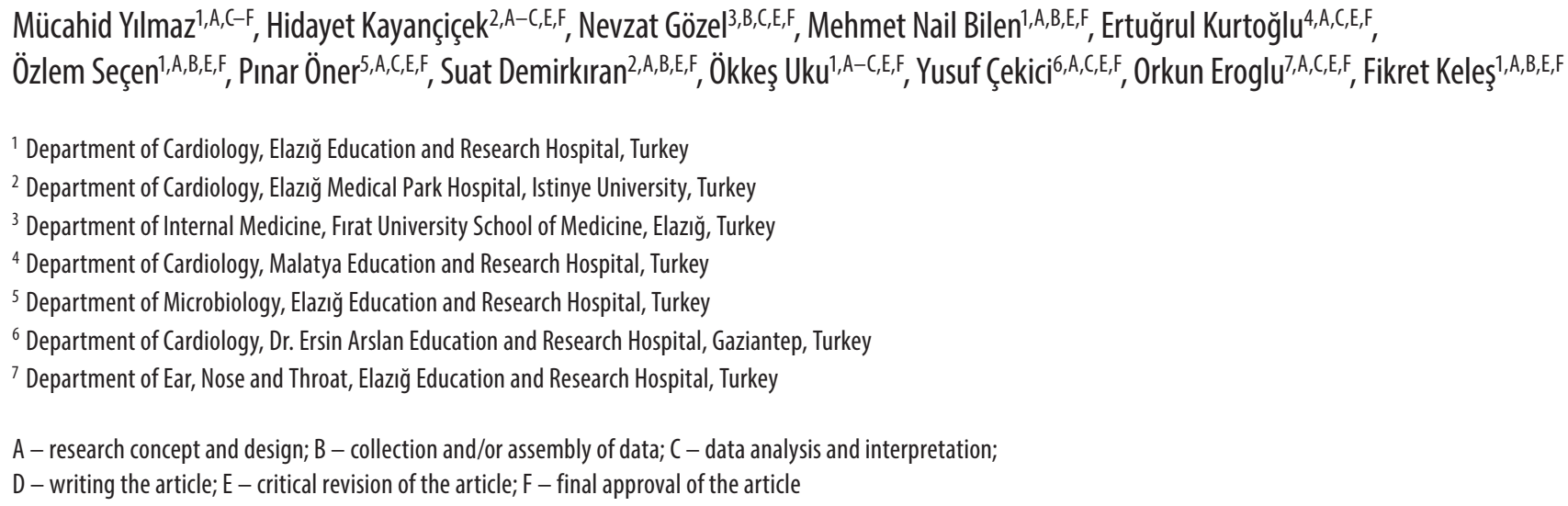

Address for correspondence

Mücahid Yılmaz

E-mail: mucahid.yilmaz@hotmail.com

Funding sources

None declared

Conflict of interest

None declared

Received on 0ctober 5, 2018

Reviewed on February 19, 2019

Accepted on January 30, 2020

Published online on September 16, 2020

Cite as

Yılmaz M, Kayançiçek H, Gözel N, et al. Spotlights on some electrocardiographic paradigms: How should we evaluate normal reference values of Tp-Te interval, Tp-Te dispersion and Tp-Te/QT ratio? Adv Clin Exp Med. 2020;29(9):1091-1099. doi:10.17219/acem/117684

DOI

10.17219/acem/117684

Copyright

Copyright by Author(s)

This is an article distributed under the terms of the

Creative Commons Attribution 3.0 Unported (CC BY 3.0)

(https://creativecommons.org/licenses/by/3.0/)

\section{Abstract}

Background. Experimental and clinical studies evaluating the Tp-Te interval and Tp-Te/QT ratio have reported conflicting data. The overlap between normal Tp-Te/QT ratios (0.17 $\pm 0.02-0.27 \pm 0.06 \mathrm{~ms}$ ) and pathological values $(0.20 \pm 0.03-0.30 \pm 0.06 \mathrm{~ms})$ measured in earlier studies has raised questions about this ECG measurement technique.

Objectives. To analyze normal values of the Tp-Te interval, Tp-Te dispersion Tp-Te(d) and the Tp-Te/QT ratio based on electrocardiographic (ECG) assessment across sex and age groups in a healthy Turkish population.

Material and methods. A total of 1,485 healthy participants (723 men) were enrolled into the study. The age of the participants ranged 17-75 years and they did not have either any cardiovascular/systemic disorders or risk factors for atherosclerosis which were detected with physical examination and laboratory tests. The Tp-Te interval, Tp-Te(d) and Tp-Te/QT ratio were determined from V1-V6 derivations.

Results. For the entire study, the median Tp-Te interval was 66.0 (64.0-70.0) ms, the Tp-Te(d) was 15.0 (10.0-20.0) ms, and the Tp-Te/QT ratio was $0.18(0.17-0.19)$. The Pearson's correlation test demonstrated that the Tp-Te/QT ratio significantly correlated with older age ( $r=0.297 ; p<0.0001)$, left ventricular (LV) end-diastolic diameter (LVEDD; $r=0.481 ; p<0.0001$ ), body mass index (BMI; $r=0.421 ; p<0.0001$ ), body surface area (BSA; $r=0.191 ; p<0.0001)$, LV end-diastolic volume (LVEDV; $r=0.484 ; p<0.0001)$, LVEDV index ( $r=0.450 ; p<0.0001)$, LV mass $(r=0.548 ; p<0.0001)$, and LV mass index $(r=0.539 ; p<0.0001)$.

Conclusions. The reference values for Tp-Te interval, Tp-Te(d) and Tp-Te/QT ratio are associated with age, BMI, BSA, LVEDV, LVEDV index, LV mass, and LV mass index. These structural elements should be considered when using these ECG parameters for assessing repolarization inhomogeneity. These findings may guide further studies assessing healthy and diseased populations.

Key words: Tp-Te interval, Tp-Te/QT ratio, Tp-Te dispersion, LV mass, LV mass index 


\section{Introduction}

Myocardial repolarization heterogeneity is considered to be sensitive to malignant ventricular arrhythmias. ${ }^{1}$ For many years, non-invasive parameters of malignant ventricular arrhythmias obtained from electrocardiograms (ECG) have been used in cases with cardiovascular disease. These parameters focus mostly on the QT distance. ${ }^{2,3}$ Dispersion of QT, which is obtained by subtracting the lowest measured QT duration from the highest measured QT duration, is also widely used in many studies and is associated with the risk of sudden death. ${ }^{4-6}$ Actually, QT dispersion is a simple, approximate evaluation of the overall heterogeneity of ventricular repolarization. ${ }^{7}$ It is also difficult to determine the normal and abnormal ranges of QT dispersion. The most important reason for this is the wide range of values reported in various studies (10-71 ms). ${ }^{8,9}$ Furtheremore, the ranges considered as normal and abnormal values were overlapped between studies and some of these values were considered as abnormal. Some authors have reported that the upper limit of QT dispersion in normal healthy individuals is $65 \mathrm{~ms}$, while others have reported that QT dispersion is a simple, approximate measurement of myocardial repolarization abnormalities and that all values proposed as the upper limit in healthy individuals are unreliable; therefore, it is possible that only the abnormal QT dispersion values $>100$ ms outside the margin of error for the calculations could have clinical importance in demonstrating repolarization abnormality. ${ }^{10,11}$ The failure to identify ECG parameters adequately and to reach a consensus on the values has spurred the search for a different assessment method for the repolarization period. ${ }^{12}$

Recent studies have identified the time from the peak of the $\mathrm{T}$ wave to the latest point of the $\mathrm{T}$ wave $(\mathrm{Tp}-\mathrm{Te}$ interval) on and ECG and the ratio of this time to the QT interval (Tp-Te/QT) as new electrocardiographic parameters for myocardial repolarization dispersion, and these parameters have been accepted as measurement methods of transmural dispersion of repolarization (TDR) which is accepted as an indicator of ventricular arrhythmias. ${ }^{13,14}$ The $\mathrm{Tp}-\mathrm{Te} / \mathrm{QT}$ ratio is not changed by fluctuations in heart rate (HR) and is more reliable for demonstrating predisposition to ventricular arrhythmia than other similar, ECG-derived indices. ${ }^{15,16}$ Gupta et al. standardized the normal value of the $\mathrm{Tp}-\mathrm{Te} / \mathrm{QT}$ ratio as $0.21 \pm 0.03$ based on measurements obtained from the V6 derivation, which reflects the left ventricular (LV) transmural axis best in healthy individuals. ${ }^{15}$ However, the overlap of normal Tp-Te/QT ratios $(0.17 \pm 0.02-0.27 \pm 0.06)$ and pathological values $(0.20 \pm 0.03-0.30 \pm 0.06)$ has raised doubts about this ECG measurement technique. ${ }^{17-21}$ To overcome reliability issues that may develop in relation to this measurement technique and to increase its applicability, we believe that the normal values can be standardized in comprehensive studies on healthy individuals with an emphasis on gender, age, body mass index (BMI), body surface area (BSA),
LV volume, LV volume index, LV mass, and LV mass index. In addition, no large-scale study has assessed the Tp-Te indices. For this reason, we aimed to conduct a prospective study to overcome these issues and provide standardized reference values for future studies.

\section{Material and methods}

\section{Study population}

This prospective study recruited 1,485 healthy participants who were admitted to the outpatient clinic of our hospital between June 2016 and January 2018. The age of the subjects who were enrolled into the study ranged from 17 to 75 years; they did not have either any cardiovascular/systemic disorders or risk factors for atherosclerosis which were detected using transthoracic echocardiography (TTE), myocardial perfusion scintigraphy or exercise stress test. The study was carried out in conformity with the Helsinki principles and ethics approval was obtained from the Presidency of T.C. Firat University Ethics Committee, Elazığ, Turkey.

The exclusion criteria were electrolyte imbalances, any drug use that might influence the QT interval (such as anti-arrhythmia agents, probucol, terfenadine, erythromycin, clarithromycin, antidepressant agents, or antipsychotic agents), pregnancy, more than 3 births, being a professional athlete, and having a BMI $>30 \mathrm{~kg} / \mathrm{m}^{2}$. In addition, the subjects who had a left or right bundle-branch block in ECG were not included in the analysis.

The blood pressure, BMI and BSA parameters were measured and recorded. The subjects who were taking antihypertensive medications, had a systolic blood pressure $\geq 140 \mathrm{~mm} \mathrm{Hg}$, and/or a diastolic blood pressure $\geq 90 \mathrm{~mm} \mathrm{Hg}$ were considered hypertensive and not included in the analysis.

The BMI and BSA were evaluated according the following formulas:

$$
\begin{gathered}
\text { BMI }=\frac{\text { weight }[\mathrm{kg}]}{\text { height }^{2}\left[\mathrm{~m}^{2}\right]} \\
\text { BSA (Mosteller formula) }=\sqrt{\text { (height }[\mathrm{cm}] \times \text { weight }[\mathrm{kg}]) / 3600}
\end{gathered}
$$

\section{Electrocardiography}

By tuning the voltage value to $10 \mathrm{~mm} / \mathrm{mV}$ and the paper speed to $50 \mathrm{~mm} / \mathrm{s}$, commonly used ECG equipment (CardiofaxV model 9320; Nihon Kohden, Tokyo, Japan) was used to record the 12-derivated ECG. After scanning the whole of the ECG recording, the intervals of the Tp-Te, the QT and the RR were evaluated. The evaluations were done with the help of a computer program which is coded in MATLAB ${ }^{\circledR}$ software (MathWorks, Natick, USA). The computer soft ware code was developed by an engineer and was dependent on the image manipulation algorithms. 


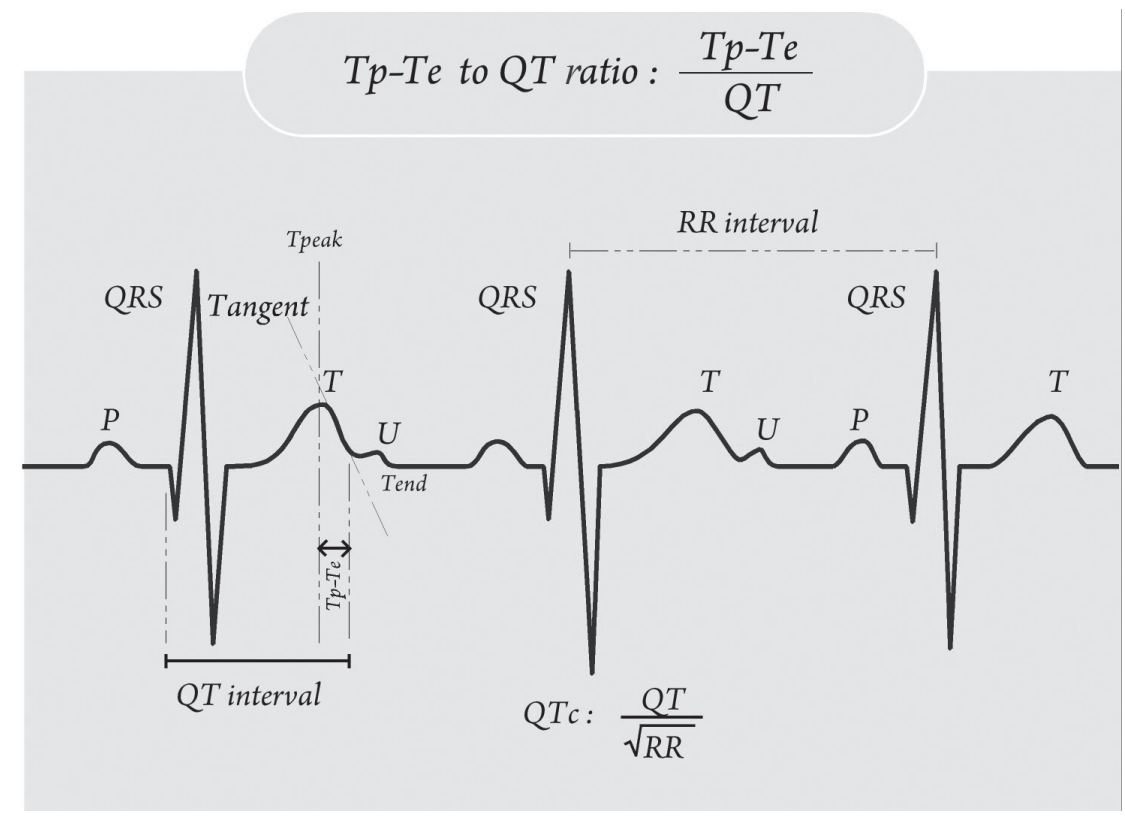

Fig. 1. Bazzett's formula and Tp-Te/QT ratio

the subsequent $\mathrm{U}$ wave). When the $\mathrm{T}$ wave was followed by a $U$ wave, the lowest point between the $T$ and $U$ waves was determined as the endpoint of the $\mathrm{T}$ wave (Fig. 1). ${ }^{24,25}$ The $\mathrm{T}$ peak was identified as the nadir of the $\mathrm{T}$ wave when negative or biphasic T waves were present (Fig. 3). ${ }^{24}$

In case of a notched $T$ wave, the endpoint of the QT distance was identified by utilizing the tangent line defined by the downward part of the $2^{\text {nd }}$ notch (Fig. 4). In the calculations, only the $\mathrm{T}$ wave amplitudes which were greater than $1.5 \mathrm{~mm}$ were included in the measurements. Otherwise they were neglected. ${ }^{25}$

All of the precordial derivations were used for the Tp-Te interval measurement

The QT interval was determined by intersecting the start value of the QRS with the final value of the $\mathrm{T}$ wave. This distance was evaluated by taking into account the $\mathrm{T}$ wave and the isoelectric line. The intersection of the tangent (drawn from the downward part of the $\mathrm{T}$ wave) and the isoelectiric line yielded the QT interval. ${ }^{22}$ Such measurements were taken from V1-V6 derivations. The QTmax values were recorded by determining the maximum values, and the QTc was determined using Bazett's formula. ${ }^{23}$ (Fig. 1). The distance between the highest peak of the $\mathrm{T}$ wave and the endpoint of the $\mathrm{T}$ wave was identified as the $\mathrm{Tp}-\mathrm{Te}$ interval (Fig. 2). The endpoint of the $\mathrm{T}$ wave was identified as the point of intersection of the tangent line and the isoelectric line (when a $U$ wave was not subsequent to the $\mathrm{T}$ wave or if the $\mathrm{T}$ wave was not distinct from

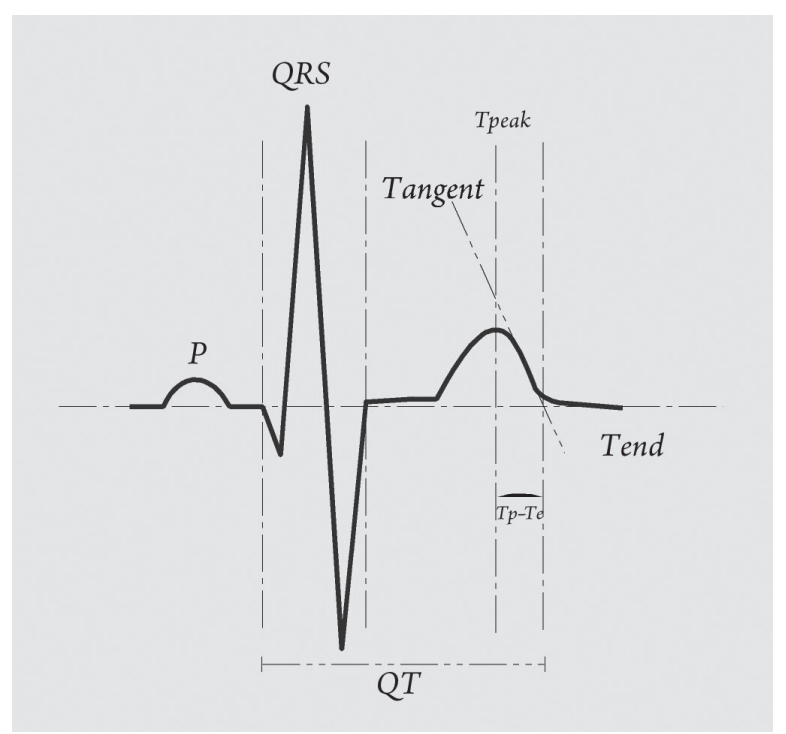

Fig. 2. The schematic presentation of the measurement of the Tp-Te and QT interval records. The highest values were accepted as the Tp-Te intervals. The $\mathrm{Tp}-\mathrm{Te}(\mathrm{d})$ values were determined by subtracting the values corresponding to the maximum and minimum Tp-Te intervals. The value of the Tp-Te/QT ratio was determined by dividing $\mathrm{Tp}-\mathrm{Te}$ to $\mathrm{QTm}$ ax values.

\section{Echocardiography}

In order to perform the transthoracic echocardiography, a Vivid 5 ultrasound machine with a $2.5-\mathrm{MHz}$ transducer (GE Medical Systems, Milwaukee, USA) was used. The American Society of Echocardiography suggestions were followed. ${ }^{26} \mathrm{M}$-mode echocardiography was used to measure interventricular septal thickness (IVS), posterior wall thickness (PW), and systolic and diastolic

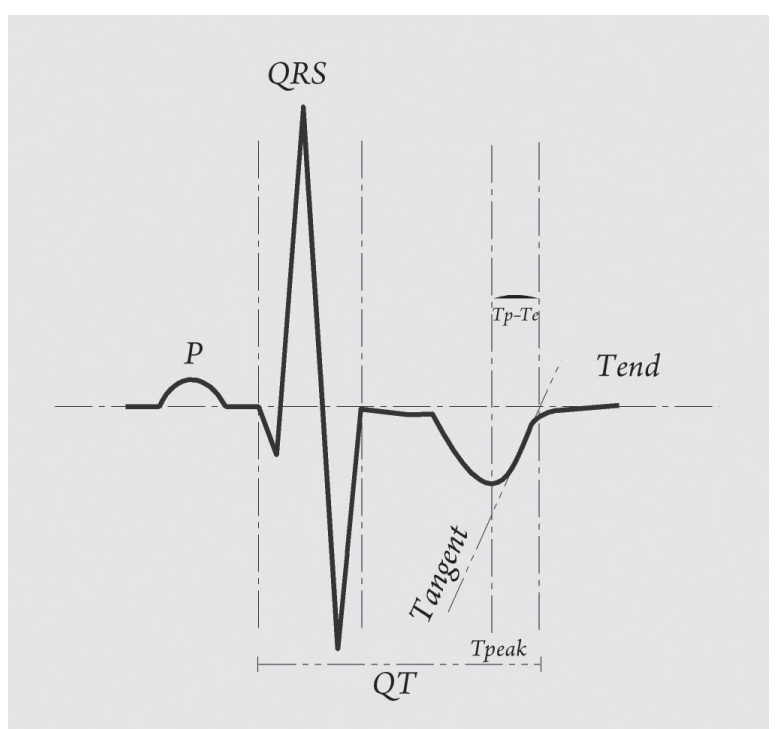

Fig. 3. The schematic presentation of the measurement of the Tp-Te interval in the presence of a negative T wave 


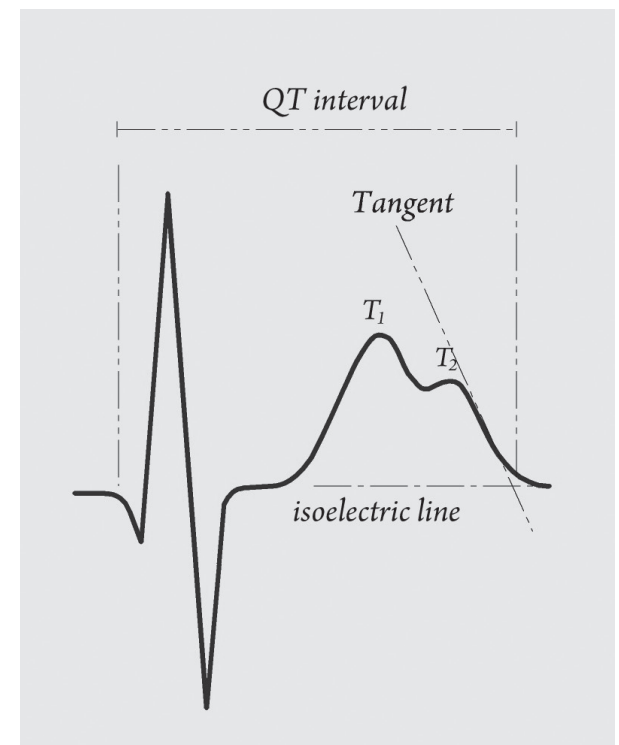

Fig. 4. Maximum slope intercept method in the presence of a notched T wave

diameters of the LV. The method that was utilized to determine the LV mass is Deveureux's formula ${ }^{27}$ :

$$
\begin{gathered}
\text { LV mass }[\mathrm{g}]= \\
=0.8 \times\left[1.04 \times\left((\text { LVEDD }+ \text { IVSd }+ \text { PWd })^{3}-(\text { LVEDD })^{3}\right)\right]+0.6
\end{gathered}
$$

where LVEDD is the LV end-diastolic diameter.

The LV mass index was computed with the help of this formula ${ }^{27}$ :

$$
\mathrm{LV} \text { mass index }\left[\mathrm{g} / \mathrm{m}^{2}\right]=\text { left ventricular mass } / \mathrm{BSA}
$$

Respectively, LVEDV (LV end-diastolic volume) and LVESV (LV end-systolic volume) values were computed as follows ${ }^{28}$ :

$$
\begin{gathered}
\operatorname{LVEDV}[\mathrm{mL}]=(7 / 2.4+\mathrm{LVEDD}) \times \text { LVEDD }^{3} \\
\operatorname{LVESV}[\mathrm{mL}]=(7 / 2.4+\mathrm{LVESD}) \times \mathrm{LVESD}^{3}
\end{gathered}
$$

The LVEDV index (LVEDVI) was computed as follows ${ }^{28}$ :

$$
\text { LVEDVI }\left[\mathrm{mL} / \mathrm{m}^{2}\right]=\left(\frac{\text { LVEDV }}{\text { BSA }}\right)
$$

LV ejection fraction (EF) and FS (fractional shortening) were determined with the help of the following formulas, respectively ${ }^{26}$ :

$$
\begin{aligned}
& \operatorname{EF~}(\%)=\left(\frac{\text { LVEDV }- \text { LVESV }}{\text { LVEDV }}\right) \times 100 \\
& \operatorname{FS~}(\%)=\left(\frac{\text { LVEDD }- \text { LVESD }}{\text { LVEDD }}\right) \times 100
\end{aligned}
$$

\section{Exercise stress test}

In order to conduct the stress test, a Cardiosis TEPA Exercise Stress Test device (TEPA Medical and Electronic Products Industry and Trade Company, Ankara, Turkey) was used. Specifically, the tests were done according to the Bruce or modified Bruce treadmill protocols. Such protocols are known to be non-invasive for functional capacity and exercise tolerance for patients who likely do not have cardiovascular disorders. ${ }^{29}$

\section{Myocardial perfusion scintigraphy}

Myocardial perfusion scintigraphy was carried out utilizing a treadmill according to Bruce or modified Bruce protocols. Sestamibi (MIBI) tagged with $10 \mathrm{mCi}$ of $99 \mathrm{mTc}$ (Cardio-Spect; Medi-Radiopharma, Budapest, Hungary) was administered intravenously to the subjects when the maximum HR (85-100\%) was reached. Gated singlephoton emission computed tomography (SPECT) imaging was taken $30 \mathrm{~min}$ later. The imaging was carried out using a GE Infinia GP3 gamma monitor system (General Electric Healthcare, Tirat Carmel, Israel) with a low-energy highresolution (LEHR) collimator. The images were evaluated using Emory Cardiac Toolbox (ECTb) myocardial quantification software (General Electric Healthcare Company).

\section{Statistical analysis}

The statistical evaluation of the results was carried out with the help of SPSS v. 16.0 (SPSS Inc., Chicago, USA) analysis program for Windows. The fit of the continuous variables to a normal distribution was analyzed with the Kolmogorov-Smirnov test. The continuous variables are presented as means with standard deviations (SD) or medians with $25^{\text {th }}-75^{\text {th }}$ percentiles. Aside from the $\mathrm{Tp}-\mathrm{Te} /$ QTc ratio and BSA $\left[\mathrm{m}^{2}\right]$, none of the continuous variables were distributed normally, and the Kolmogorov-Smirnov test was used to evaluate these variables. A descriptive statistics test was used to evaluate the Tp-Te/QTc ratio and BSA $\left[\mathrm{m}^{2}\right]$. These values are presented as means \pm SD. All other data is presented as medians with $25^{\text {th }}-75^{\text {th }}$ percentiles. Pearson's correlation test was used to perform correlation analyses. A value of $\mathrm{p}<0.05$ was required for statistical significance.

\section{Results}

Table 1 summarizes the baseline features of the participants. The study involved 1,485 healthy subjects (723 men and 762 women). The age of all participants, male participants and female participants was 42.0 years (30.0-53.5), 41.0 years (30.0-52.0) and 43.5 years (30.0-55.0), respectively. Table 1 also lists the findings for the ventricular repolarization parameters. The median Tp-Te interval was $66.0 \mathrm{~ms}(64.0-70.0)$, the $\mathrm{Tp}-\mathrm{Te} / \mathrm{QT}$ ratio was 0.18 (0.17-0.19), the mean Tp-Te/QTc ratio was $0.16 \pm 0.014$, and the median Tp-Te(d) was $15.0 \mathrm{~ms}$ (10.0-20.0) for all participants.

The results revealed that the $\mathrm{Tp}-\mathrm{Te} / \mathrm{QT}$ ratio significantly correlated with age, PW, IVS, LVEDD, BMI, BSA, 
Table 1. Clinical characteristics of the study population

\begin{tabular}{|c|c|c|c|}
\hline Parameters & $\begin{array}{l}\text { All participants } \\
\qquad n=1,485\end{array}$ & $\begin{array}{c}\text { Men } \\
n=723\end{array}$ & $\begin{array}{l}\text { Women } \\
n=762\end{array}$ \\
\hline Age & $42.0(30.0-53.5)$ & $41.0(30.0-52.0)$ & $43.5(30.0-55.0)$ \\
\hline Tp-Te [ms] & $66.0(64.0-70.0)$ & $66.0(63.0-70.0)$ & $67.0(64.0-70.0)$ \\
\hline QTmax [ms] & $361.0(353.5-371.0)$ & $360.0(352.0-370.0)$ & $362.0(355.0-372.0)$ \\
\hline QTc [ms] & 398.73 (383.02-417.79) & 393.56 (380.0-410.79) & $402.77(387.60-422.62)$ \\
\hline Tp-Te/QT ratio & $0.18(0.17-0.19)$ & $0.18(0.17-0.19)$ & $0.18(0.18-0.19)$ \\
\hline Tp-Te/QTc ratio\# & $0.16 \pm 0.014$ & $0.17 \pm 0.015$ & $0.16 \pm 0.014$ \\
\hline Tp-Te(d) [ms] & $15.0(10.0-20.0)$ & $14.0(10.0-18.0)$ & $15.0(10.0-20.0)$ \\
\hline$H R$ & $74.0(69.0-80.0)$ & $71.0(68.0-78.0)$ & $75.0(70.0-80.0)$ \\
\hline $\mathrm{PW}[\mathrm{mm}]$ & $8.0(7.0-9.0)$ & $8.0(7.0-9.0)$ & $7.5(7.0-8.0)$ \\
\hline IVS [mm] & $8.0(7.0-9.0)$ & $8.0(8.0-9.0)$ & $8.0(7.0-9.0)$ \\
\hline LVEDD [mm] & $44.0(42.0-46.0)$ & $45.0(43.0-47.0)$ & $42.5(40.0-45.0)$ \\
\hline Length [m] & $1.68(1.62-1.74)$ & $1.74(1.71-1.77)$ & $1.62(1.59-1.65)$ \\
\hline Weight [kg] & $75.0(67.0-81.0)$ & $79.0(74.0-84.0)$ & $68.0(63.0-72.0)$ \\
\hline $\mathrm{BMI}\left[\mathrm{kg} / \mathrm{m}^{2}\right]$ & $26.10(24.61-27.58)$ & $26.12(24.69-27.66)$ & $26.08(24.53-27.58)$ \\
\hline $\mathrm{BSA}\left[\mathrm{m}^{2}\right]^{\#}$ & $1.84 \pm 0.16$ & $1.95 \pm 0.11$ & $1.74 \pm 0.12$ \\
\hline LVEDV [mL] & 87.68 (78.57-97.33) & $92.44(83.06-102.36)$ & 80.82 (70.00-92.44) \\
\hline LVEDV index [mL/m²] & $46.44(42.40-51.45)$ & $46.55(42.88-51.04)$ & $46.37(41.94-51.92)$ \\
\hline EF [\%] & $64.84(62.14-68.11)$ & $63.69(61.04-66.30)$ & $66.30(63.26-69.41)$ \\
\hline FS [\%] & $35.0(33.33-37.5)$ & 34.69 (32.60-36.36) & $36.36(34.09-38.46)$ \\
\hline LV mass [g] & 105.92 (89.97-132.92) & 114.76 (99.92-138.32) & 97.38 (82.49-119.17) \\
\hline LV mass index $\left[\mathrm{g} / \mathrm{m}^{2}\right]$ & 57.55 (50.12-69.49) & 59.33 (52.12-71.37) & 55.33 (48.85-67.51) \\
\hline
\end{tabular}

BMI - body mass index; BSA - body surface area; EF - ejection fraction; FS - fractional shortening; HR - heart rate; IVS - interventricular septum; LV - left ventricle; LVEDD - left ventricular end-diastolic diameter; LVESD - left ventricular end-systolic diameter; LVEDV - left ventricular end-diastolic volume; LVESV - left ventricular end-systolic volume; PW - posterior wall; QTmax - QT maximum; QTc - QT corrected. \# No other continuous values - except $\mathrm{Tp}-\mathrm{Te} / \mathrm{QTc}$ ratio and BSA [m²] - were normally distributed; the Kolmogorov-Smirnov test was used to evaluate these variables. A descriptive statistics test was used to evaluate Tp-Te/QTc ratio and BSA [m²].

Table 2. Ventricular repolarization parameters across age subgroups

\begin{tabular}{|c|c|c|c|c|}
\hline Age subgroups (n) & Tp-Te [ms] & $\mathrm{Tp}-\mathrm{Te} / \mathrm{QT}$ ratio & $\mathrm{Tp}-\mathrm{Te} / \mathrm{QTc}$ ratio & $\mathrm{Tp}-\mathrm{Te}(\mathrm{d})[\mathrm{ms}]$ \\
\hline 10-19 years (116) & $60.0(58.0-63.0)$ & $0.17(0.16-0.18)$ & $0.15(0.14-0.16)$ & $10.0(5.0-10.0)$ \\
\hline $20-29$ years (237) & $63.0(60.0-66.0)$ & $0.18(0.17-0.19)$ & $0.16(0.15-0.17)$ & $10.0(10.0-15.0)$ \\
\hline 30-39 years (322) & $66.0(63.0-68.0)$ & $0.18(0.17-0.18)$ & $0.16(0.15-0.17)$ & $12.0(10.0-16.0)$ \\
\hline $40-50$ years (317) & $67.0(66.0-69.5)$ & $0.18(0.18-0.19)$ & $0.17(0.16-0.18)$ & $15.0(10.0-17.5)$ \\
\hline 50-59 years (311) & $70.0(67.0-70.0)$ & $0.18(0.18-0.19)$ & $0.17(0.16-0.18)$ & $18.0(10.0-20.0)$ \\
\hline 60-69 years (138) & $70.0(70.0-75.0)$ & $0.19(0.18-0.20)$ & $0.17(0.16-0.18)$ & $20.0(15.75-25.0)$ \\
\hline $70-79$ years (44) & $75.0(70.0-80.0)$ & $0.19(0.19-0.20)$ & $0.18(0.17-0.18)$ & $20.0(20.0-29.0)$ \\
\hline
\end{tabular}

LVEDV, LVEDV index, LV mass, and LV mass index. Additionally, significant correlations were found between age and BMI $(\mathrm{r}=0.416 ; \mathrm{p}<0.0001)$, BSA $(\mathrm{r}=0.101 ; \mathrm{p}<0.0001)$, LVEDV index $(r=0.394 ; \mathrm{p}<0.0001)$, and LV mass index $(\mathrm{r}=0.484 ; \mathrm{p}<0.0001)$.

\section{Discussion}

The present study reports normal ECG TDR parameters by measuring the Tp-Te interval, Tp-Te(d) and the Tp-Te/ QT ratio in a healthy population without any systemic/ cardiovascular disorders or risk factors for atherosclerosis (Tables 1 and 2). We demonstrated that there is a significant correlation between the TDR parameters and echocardiographic measurements (Table 3; Fig. 6). Notably, the TDR parameters increased with older age (Tables 2 and 3; Fig. 5).

Although the QT interval and QT dispersion measurements are important ECG predictors of ventricular arrhythmogenesis, some studies have questioned their prognostic importance. ${ }^{30}$ The failure to adequately identify ECG parameters and to reach a consensus on the values identified - which is due to a variety of reasons - have spurred the search for a different assessment method for 
Table 3. Pearson correlation analysis between ventricular repolarization parameters and echocardiographic parameters

\begin{tabular}{|c|c|c|c|c|c|c|c|c|}
\hline \multirow{2}{*}{ Parameters } & \multicolumn{2}{|c|}{$\mathrm{Tp}-\mathrm{Te}[\mathrm{ms}]$} & \multicolumn{2}{|c|}{ Tp-Te/QT ratio } & \multicolumn{2}{|c|}{ Tp-Te/QTc ratio } & \multicolumn{2}{|c|}{$\mathrm{Tp}-\mathrm{Te}(\mathrm{d})[\mathrm{ms}]$} \\
\hline & $r$ & $\mathrm{p}$-value & $r$ & $\mathrm{p}$-value & $r$ & $\mathrm{p}$-value & $r$ & p-value \\
\hline Age & 0.583 & $<0.0001$ & 0.297 & $<0.0001$ & 0.350 & $<0.0001$ & 0.476 & $<0.0001$ \\
\hline $\mathrm{PW}[\mathrm{mm}]$ & 0.631 & $<0.0001$ & 0.464 & $<0.0001$ & 0.414 & $<0.0001$ & 0.486 & $<0.0001$ \\
\hline IVS [mm] & 0.641 & $<0.0001$ & 0.467 & $<0.0001$ & 0.415 & $<0.0001$ & 0.505 & $<0.0001$ \\
\hline LVEDD $[\mathrm{mm}]$ & 0.578 & $<0.0001$ & 0.481 & $<0.0001$ & 0.424 & $<0.0001$ & 0.460 & $<0.0001$ \\
\hline BMI $\left[\mathrm{kg} / \mathrm{m}^{2}\right]$ & 0.520 & $<0.0001$ & 0.421 & $<0.0001$ & 0.406 & $<0.0001$ & 0.394 & $<0.0001$ \\
\hline $\mathrm{BSA}\left[\mathrm{m}^{2}\right]$ & 0.204 & $<0.0001$ & 0.191 & $<0.0001$ & 0.213 & $<0.0001$ & 0.136 & $<0.0001$ \\
\hline LV mass [g] & 0.708 & $<0.0001$ & 0.548 & $<0.0001$ & 0.484 & $<0.0001$ & 0.560 & $<0.0001$ \\
\hline LV mass index $\left[\mathrm{g} / \mathrm{m}^{2}\right]$ & 0.712 & $<0.0001$ & 0.539 & $<0.0001$ & 0.458 & $<0.0001$ & 0.570 & $<0.0001$ \\
\hline LVEDV [mL] & 0.579 & $<0.0001$ & 0.484 & $<0.0001$ & 0.425 & $<0.0001$ & 0.463 & $<0.0001$ \\
\hline LVEDV index $\left[\mathrm{mL} / \mathrm{m}^{2}\right]$ & 0.550 & $<0.0001$ & 0.450 & $<0.0001$ & 0.366 & $<0.0001$ & 0.453 & $<0.0001$ \\
\hline
\end{tabular}

BMI - body mass index; BSA - body surface area; IVS - interventricular septal thickness; LV mass - left ventricular mass; LVEDD - left ventricular end-diastolic diameter; LVEDV - left ventricular end-diastolic volume; PW - posterior wall thickness.

Table 4. Pearson correlation analysis between age and BMI, BSA, LVEDV index, and LV mass index

\begin{tabular}{|c|c|c|c|c|c|c|c|c|}
\hline \multirow{2}{*}{ Parameters } & \multicolumn{2}{|c|}{ BMI $\left[\mathrm{kg} / \mathrm{m}^{2}\right]$} & \multicolumn{2}{|c|}{$\mathrm{BSA}\left[\mathrm{m}^{2}\right]$} & \multicolumn{2}{|c|}{ LVEDV index $\left[\mathrm{mL} / \mathrm{m}^{2}\right]$} & \multicolumn{2}{|c|}{ LV mass index $\left[\mathrm{g} / \mathrm{m}^{2}\right]$} \\
\hline & r & $p$-value & $r$ & $\mathrm{p}$-value & $r$ & $p$-value & $r$ & $p$-value \\
\hline Age & 0.416 & $<0.0001$ & 0.101 & $<0.0001$ & 0.394 & $<0.0001$ & 0.484 & $<0.0001$ \\
\hline
\end{tabular}

the repolarization period. ${ }^{31}$ The JT interval (the distance from the endpoint of the QRS structure to the endpoint of the $\mathrm{T}$ wave), the QT apex interval (the distance from the starting point of the QRS complex to the peak point of the $\mathrm{T}$ wave: QTa), and the $\mathrm{T}$ wave area (the total area below the J-point and the endpoint of the $\mathrm{T}$ wave) are examples of this search. ${ }^{32}$ However, these novel repolarization parameters are no more accurate than conventional QT dispersion in assessing dispersion, and in some cases did not correlate with QT dispersion. ${ }^{32,33}$

In recent studies, the Tp-Te interval has been accepted as a TDR index. ${ }^{13,14}$ However, variations in HR and body weight may affect the Tp-Te interval. ${ }^{15}$ Ventricular myocardium is an electrically inhomogeneous construction

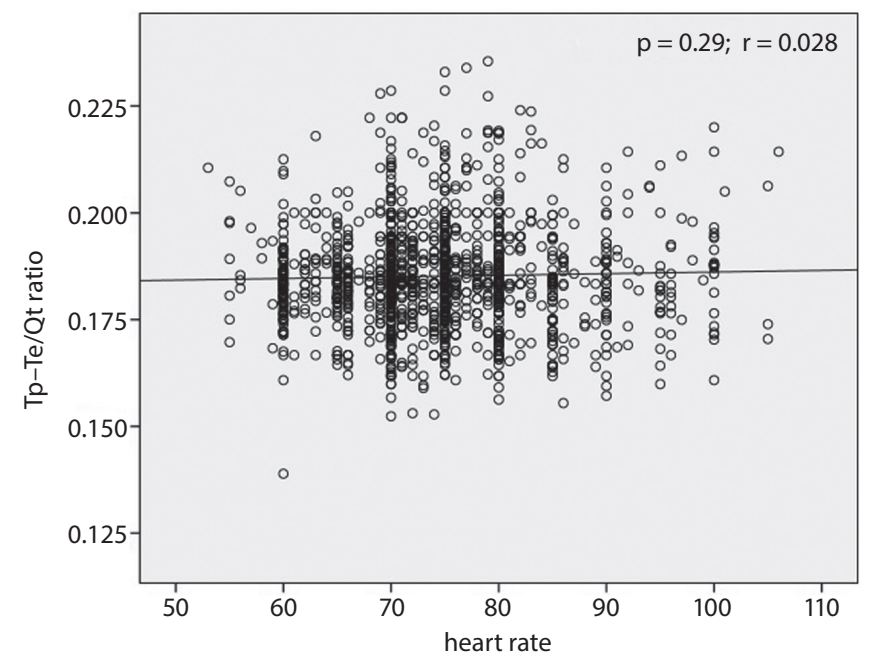

Fig. 5. The correlations between age, heart rate and Tp-Te/QT ratio and is composed of 3 different cell types: epicardial, endocardial and $\mathrm{M}$ cells. The $\mathrm{M}$ cells (masonic midmyocardial Moe cells) are settled into the subendocardial lamina. These cells have a longer period of action potential than epicardial or endocardial cells when exposed to heart rate decelerating agents. ${ }^{15}$ The period of action potential (PAP) is the longest in M cells. Among these 3 cell types, the $M$ cells have the lowest, most slowly activating retarded potassium current (IKs) but the highest, late sodium current (INa). On the other hand, the density of the rapidly activating retarded potassium current (IKr) does not differ among the 3 layers. Thus, the discrepancy at the IKs/IKr ratio among the ventricular myocardial layers plays an important role in the transmural dispersion of TDR. ${ }^{33}$

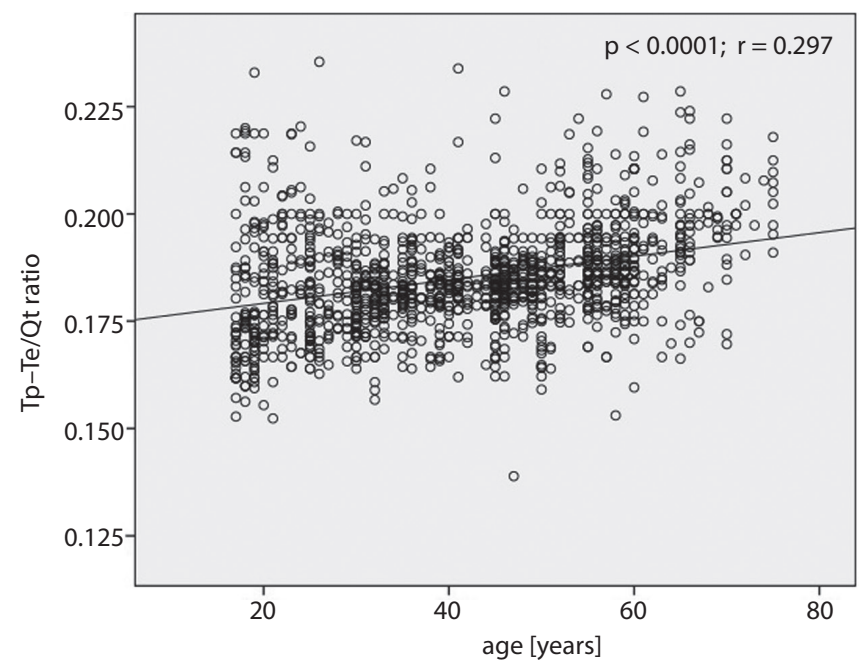



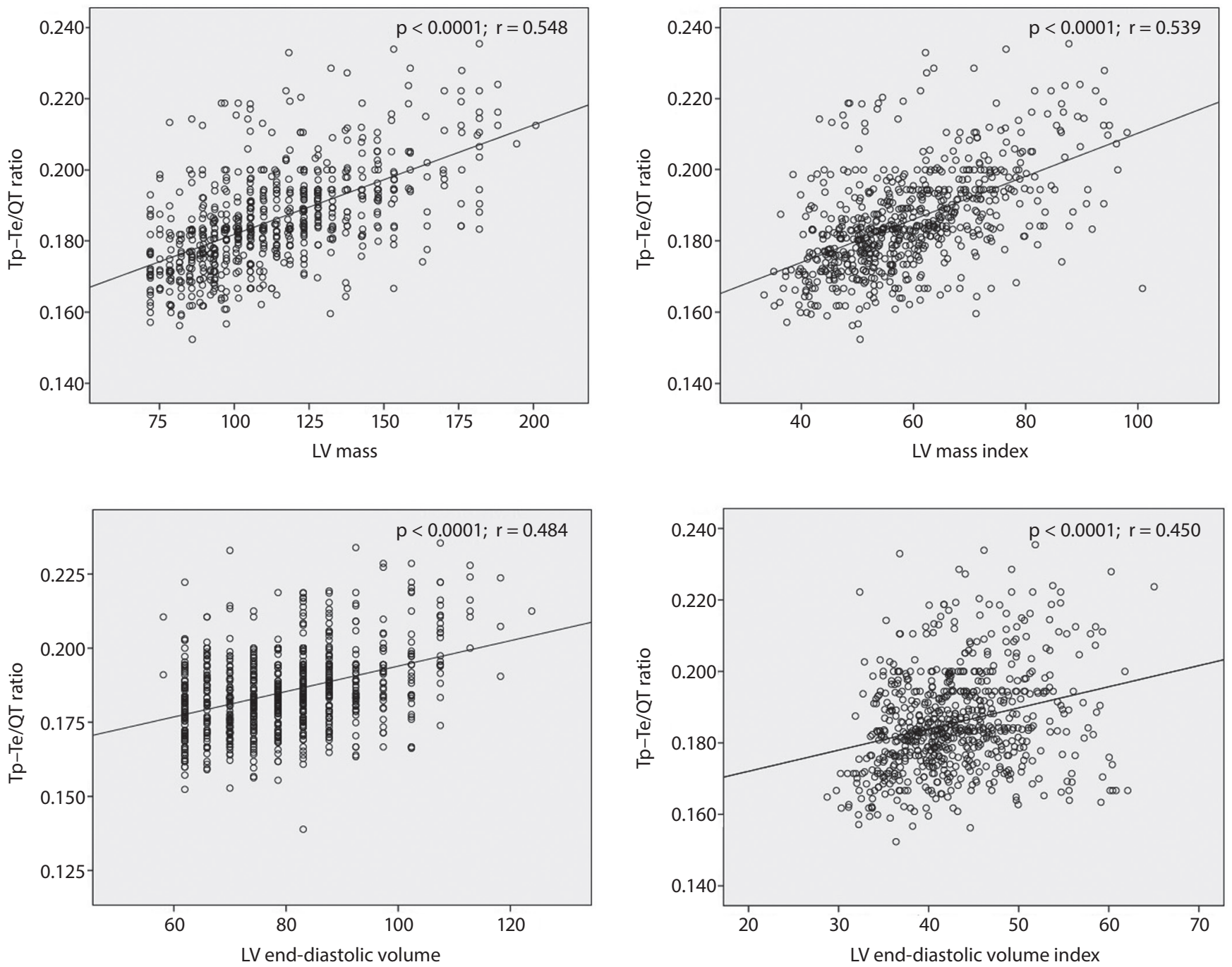

Fig. 6. The correlations between LV mass, LV mass index, LV volume, LV volume index, and Tp-Te/QT ratio

The epicardial cells complete their repolarization earlier than the other 2 cells. The highest point of the $\mathrm{T}$ wave (Tp) reflects the end of the epicardial PAP, and the final point of the $\mathrm{T}$ wave (Te) reflects the end of the midmyocardial PAP. Consequently, the Tp-Te duration is accepted as a representation of the TDR. ${ }^{17}$ However, variations in HR might affect the Tp-Te duration. ${ }^{15}$

Recently, the Tp-Te/QT ratio has been accepted as a new, non-invasive ECG marker of ventricular repolarization inhomogeneity, and it has been suggested that it may be used as an accurate parameter of ventricular repolarization heterogeneity independent of changes in HR. ${ }^{15,16}$ Gupta et al. also suggested that the $\mathrm{Tp}-\mathrm{Te} / \mathrm{QT}$ ratio is a more accurate indicator of ventricular arrhythmogenesis when compared with the QT, QTc and Tp-Te durations. ${ }^{15}$ However, there are also conflicting reports on normal and abnormal values for these parameters. ${ }^{1,17-21}$

The present study demonstrated that the HR of healthy subjects varied from $53 \mathrm{bpm}$ to $106 \mathrm{bpm}$. The Tp-Te duration diminished in parallel with an increase in HR, with the $\mathrm{Tp}-\mathrm{Te}$ duration ranging from $50 \mathrm{~ms}$ to $86 \mathrm{~ms}$
(66.0 (64.0-70.0) ms). Likewise, the Tp-Te/QTc ratio and $\mathrm{Tp}-\mathrm{Te}(\mathrm{d})$ values diminished in parallel with increased HR. On the other hand, in spite of the alterations in HR, the $\mathrm{Tp}-\mathrm{Te} / \mathrm{QT}$ ratio remained stable with a median of 0.18 (0.17-0.19) (Fig. 5). The results suggest that the Tp-Te/Qt ratio may be more appropriate than other parameters for assessing TDR.

Recent studies have demonstrated that risk factors for atherosclerosis, such as diabetes mellitus, hypertension and smoking - in addition to structural heart diseases and myocardial ischemia - give rise to an increase in ventricular repolarization parameters (TDR) and the heterogeneity of ventricular repolarization. ${ }^{1,16,17,19,23}$ Furthermore, a number of studies have stated that LV enlargement and increased LV mass may be helpful indices for more ventricular arrhythmia risk stratification. ${ }^{34-36}$ It has been reported that TDR parameters may increase with older age because of aging processes taking place in the myocardium, such as fibrosis. ${ }^{37}$ Another explanation for the increased TDR with aging is distortion of the balance between sympathetic and parasympathetic tone in favor of sympathetic 
Table 5. Pearson correlation analysis between BMI, BSA, LV mass, and LV mass index

\begin{tabular}{|l|c|c|c|c|}
\multirow{2}{*}{\multicolumn{1}{|c|}{ Parameters }} & \multicolumn{2}{|c|}{ LV mass } & \multicolumn{2}{c|}{ LV mass index } \\
\cline { 2 - 5 } & $\mathrm{r}$ & $\mathrm{p}$-value & $\mathrm{r}$ & $\mathrm{p}$-value \\
\hline $\mathrm{BMI}\left[\mathrm{kg} / \mathrm{m}^{2}\right]$ & 0.447 & $<0.0001$ & 0.265 & $<0.0001$ \\
\hline $\mathrm{BSA}\left[\mathrm{m}^{2}\right]$ & 0.484 & $<0.0001$ & 0.159 & $<0.0001$ \\
\hline
\end{tabular}

tone. In old age, this balance shifts substantially towards the sympathetic activity. ${ }^{38}$ Increased sympathetic activity can cause several changes in myocardial membrane characteristics which cause early depolarizations and increased TDR. ${ }^{39-41}$ Our study shows a relationship between age, BMI, BSA, and TDR parameters (Table 3). Although the mechanisms underlying such a relationship are not wellknown, it is possible that an increase in sympathetic activity, BMI and BSA may lead to an increase in cardiac workload in these subjects, and this situation might be responsible for the increased LV mass and LV mass index (Tables 4 and 5).

Overlapping with previous research, the current study - which excludes myocardial ischemia and risk factors for atherosclerosis as well as systemic and structural heart disease - shows that BMI, BSA, age, and echocardiographic measurements have a direct effect on TDR parameters. The study demonstrated that the values of BMI, BSA, PW, IVS, LVEDD, BSA, LVEDV, LVEDV index, LV mass, and LV mass index among Turkish people increase with age; these findings significantly correlated with an elevated Tp-Te/ QT ratio, suggesting an increased risk of ventricular arrhythmia (Tables 2-4; Fig. 6). More clearly, the LV mass and LV mass index values of Turkish people increase with age, and the $\mathrm{Tp}-\mathrm{Te} / \mathrm{QT}$ ratio increases along with these values (Tables 2-4). In other words, our data implies that aging and elevated BMI and BSA may cause increased ventricular repolarization heterogeneity via ventricular structural remodeling.

It is reasonable to suppose that the increase in myocardial mass, which leads to increased LV mass and LV mass index, may be caused by a further proportional increase in $M$ cells, with the knowledge that $M$ cells contribute more to repolarization time than epicardial and endocardial cells. However, an invasive method such as biopsy is required to enable a definitive evaluation of this link.

\section{Conclusions}

The LV mass and LV mass index increase with age independently of the individual's health, and these parameters cause TDR values to increase. It is more rational to use the $\mathrm{Tp}-\mathrm{Te} / \mathrm{QT}$ ratio for TDR measurements because it is the least affected by HR. Future studies using the Tp$\mathrm{Te} / \mathrm{QT}$ ratio should evaluate the parameters of age, BMI, PW, IVS, LVEDD, BSA, LVEDV, LVEDV index, and LV mass and LV mass index in patient and control groups, and even if the individual appears healthy, it should be considered in other situations (race, pregnancy, professions requiring intensive labor - such as porterage and construction work - being a professional athlete, etc.) that may affect the individual's LV mass and LV mass index.

\section{ORCID iDs}

Mücahid Yılmaz (D) https://orcid.org/0000-0003-1458-8637 Hidayet Kayançiçek (D) https://orcid.org/0000-0001-6493-5591 Nevzat Gözel (1D https://orcid.org/0000-0001-7326-6860 Mehmet Nail Bilen (D) https://orcid.org/0000-0003-1468-2930 Ertuğrul Kurtoğlu (D) https://orcid.org/0000-0001-5835-087X Özlem Seçen (i) https://orcid.org/0000-0003-4657-8003 Pınar Öner (10) https://orcid.org/0000-0001-9592-5986 Suat Demirkıran (D) https://orcid.org/0000-0002-6769-8875 Ökkeş Uku (D) https://orcid.org/0000-0002-4392-7522

Yusuf Çekici (D) https://orcid.org/0000-0002-4585-3707

Orkun Eroglu (D) https://orcid.org/0000-0001-9392-5755

Fikret Keleş (D) https://orcid.org/0000-0003-1012-3875

\section{References}

1. Karaagac K, Yontar OC, Tenekecioglu E, et al. Evaluation of Tp-Te interval and Tp-Te/QTc ratio in patients with coronary artery ectasia. Int J Clin Exp Med. 2014;7(9):2865-2870.

2. Al-Zaiti SS, Fallavollita JA, Canty Jr JM, Carey MG. Electrocardiographic predictors of sudden and non-sudden cardiac death in patients with ischemic cardiomyopathy. Heart Lung. 2014;43(6):527-533.

3. Schouten E, Dekker J, Meppelink P, Kok F, Vandenbroucke J, Pool J. Qt interval prolongation predicts cardiovascular mortality in an apparently healthy population. Circulation. 1991;84(4):1516-1523.

4. Di lorio B. Relevance of qt dispersion in haemodialysis patients. Nephrol Dial Transpl. 2010;25(4):1357-1360.

5. Malhis M, Al-Bitar S, Farhood S, Zaiat K. Changes in QT intervals in patients with end-stage renal disease before and after hemodialysis. Saudi J Kidney Dis Transpl. 2010;21(3):460-465.

6. Genovesi S, Dossi C, Vigano MR, et al. Electrolyte concentration during haemodialysis and QT interval prolongation in uraemic patients. Europace. 2008;10(6):771-777.

7. Ünal S, Yayla Ç, Açar B, et al. Tp-e interval and Tp-e/QT ratio in patients with human immunodeficiency virus. J Infect Public Heal. 2018;11(1): 35-38.

8. Malik M, Batchvarov VN. Measurement, interpretation and clinical potential of QT dispersion. J Am Coll Cardiol. 2000;36(6):1749-1766.

9. Punske BB, Lux RL, MacLeod RS, et al. Mechanisms of the spatial distribution of QT intervals on the epicardial and body surfaces. J Cardiovasc Electrophysiol.1999;10(12):1605-1618.

10. Surawicz B. Will QT dispersion play a role in clinical decision-making? J Cardiovasc Electrophysiol. 1996;7:777-784.

11. Çelik T, İyisoy A, Çelik M, Işık E. The clinical significance of QT dispersion: How much reliable and beneficial? Arch Turk Soc Cardiol. 2007; 35(8):510-511.

12. Fak AS, Tezcan H, Oktay A. QT dispersion: An interesting research field, or a useful diagnostic tool? Arch Turk Soc Cardiol. 2000;28:51-59.

13. Kors JA, van Eck HJR, van Herpen G. The meaning of the Tp-Te interval and its diagnostic value. J Electrocardiol. 2008;41(6):575-580.

14. Antzelevitch C, Sicouri S, Di Diego JM, et al. Does Tpeak-Tend provide an index of transmural dispersion of repolarization? Heart Rhythm. 2007:4(8):1114-1116.

15. Gupta $P$, Patel C, Patel $H$, et al. Tp-e/QT ratio as an index of arrhythmogenesis. J Electrocardiol. 2008;41(6):567-574.

16. Zhao X, Xie Z, ChuY, et al. Association between Tp-e/QT ratio and prognosis in patients undergoing primary percutaneous coronary intervention for ST-segment elevation myocardial infarction. Clin Cardiol. 2012;35(9):559-564.

17. Tokatli A, Kiliçaslan F, Alis M, Yiginer O, Uzun M. Prolonged Tp-e interval, Tp-e/QT ratio and Tp-e/QTc ratio in patients with type 2 diabetes mellitus. Endocrinol Metab (Seoul). 2016;31(1):105-112.

18. Karaagac K, Emul A, Tenekecioglu E, et al. The effects of metabolic syndrome on Tp-Te interval and Tp-Te/QT ratio in patients with normal coronary arteries. Eurasian J Med. 2014;46(3):182-186. 
19. Taşolar H, Ballı M, Bayramoğlu A, et al. Effect of smoking on Tp-e interval, Tp-e/QT and Tp-e/QTc ratios as indices of ventricular arrhythmogenesis. Heart Lung Circ. 2014;23(9):827-832.

20. Ilgenli TF, Tokatlı A, Akpınar O, Kılıçaslan F. The effects of cigarette smoking on the Tp-e interval, Tp-e/QT ratio and Tp-e/QTc ratio. Adv Clin Exp Med. 2015;24(6):973-978.

21. Karaman $\mathrm{K}$, Altunkaş $\mathrm{F}$, Cetin $\mathrm{M}$, et al. New markers for ventricular repolarization in coronary slow flow: Tp-e interval, Tp-e/QT ratio, and Tp-e/QTc ratio. Ann Noninvasive Electrocardiol. 2015;20(4):338-344.

22. Hevia JC, Antzelevitch C, Bárzaga FT, et al. Tpeak-Tend and TpeakTend dispersion as risk factors for ventricular tachycardia/ventricular fibrillation in patients with the Brugada syndrome. J Am Coll Cardiol. 2006;47(9):1828-1834.

23. Yontar OC, Karaagac K, Tenekecioglu E, Tutuncu A, Demir M, Melek M. Assessment of ventricular repolarization inhomogeneity in patients with mitral valve prolapse: Value of T wave peak to end interval. Int J Clin Exp Med. 2014;7(8):2173-2178.

24. Icli A, Kayrak M, Akilli H, et al. Prognostic value of Tpeak-Tend interval in patients with acute pulmonary embolism. BMC Cardiovasc Disord. 2015;15:99.

25. Burns E. ECG Basics: Waves, Intervals, Segments and Clinical Interpretation. https://lifeinthefastlane.com > ECG Library. Accessed May 20, 2018.

26. Lang RM, Bierig M, Devereux RB, et al. Recommendations for chamber quantification. Eur J Echocardiogr. 2006;7:79-108.

27. Lang RM, Bierig M, Devereux RB, et al. Recommendations for chamber quantification: A report from the American Society of Echocardiography's Guidelines and Standards Committee and the Chamber Quantification Writing Group, developed in conjunction with the European Association of Echocardiography, a branch of the European Society of Cardiology.. J Am Soc Echocardiogr. 2005;18(12):1440-1463.

28. Hudsmith LE, Petersen SE, Francis JM, Robson MD, Neubauer S. Normal human left and right ventricular and left atrial dimensions using steady state free precession magnetic resonance imaging. J Cardiovasc Magn Reson. 2005;7(5):775-782.
29. Bruce R, Blackmon J, Jones J, Strait G. Exercising testing in adult normal subjects and cardiac patients. Ann Noninvasive Electrocardiol. 2004;9(3):291-303.

30. Pueyo E, Martínez JP, Laguna P. Cardiac repolarization analysis using the surface electrocardiogram. Philos Trans A Math Phys Eng Sci. 2009; 367(1887):213-233.

31. Kaya D, Guler C, Esen AM, Barutcu I, Dincel C. Sildenafil citrate does not alter ventricular repolarization properties: Novel evidence from dynamic QT analysis. Ann Noninvasive Electrocardiol. 2004;9(3):228-233.

32. Zabel M, Klingenheben T, Franz MR, Hohnloser SH. Assessment of QT dispersion for prediction of mortality or arrhythmic events after myocardial infarction: Results of a prospective, long-term follow-up study. Circulation. 1998;97(25):2543-2550.

33. Yan G-X, Lankipalli RS, Burke JF, Musco S, Kowey PR. Ventricular repolarization components on the electrocardiogram: Cellular basis and clinical significance. J Am Coll Cardiol. 2003;42(3):401-409.

34. Haider AW, Larson MG, Benjamin EJ, Levy D. Increased left ventricular mass and hypertrophy are associated with increased risk for sudden death. J Am Coll Cardiol. 1998;32(5):1454-1459.

35. Aleong RG, Mulvahill MJ, Halder I, et al. Left ventricular dilatation increases the risk of ventricular arrhythmias in patients with reduced systolic function. J Am Heart Assoc. 2015;4(8):e001566.

36. Narayanan K, Reinier K, Teodorescu C, et al. Left ventricular diameter and risk stratification for sudden cardiac death. J Am Heart Assoc. 2014;3(5):e001193.

37. Reardon M, Malik M. Qt interval change with age in an overtly healthy older population. Clin Cardiol. 1996;19(12):949-952.

38. Pfeifer MA, Weinberg CR, Cook D, Best JD, Reenan A, Halter JB. Differential changes of autonomic nervous system function with age in man. Am J Med. 1983;75(2):249-258.

39. Zipes DP. The long QT interval syndrome: A Rosetta Stone for sympathetic related ventricular tachyarrhythmias. Circulation. 1991;84(3): 1414-1419.

40. Ben-David J, Zipes DP. Differential response to right and left ansae subclaviae stimulation of early afterdepolarizations and ventricular tachycardia induced by cesium in dogs. Circulation. 1988;78:1241-1250. 